\title{
Right-ear advantage and delayed recall
}

\author{
JOHN NEIL BOHANNON III \\ Georgia Institute of Technology, Atlanta, Georgia 30332 \\ and \\ LYNN BAKER-WARD \\ University of North Carolina, Chapel Hill, North Carolina 27514
}

\begin{abstract}
Two studies were performed using monaural presentation of verbal material to test for a right-ear advantage (REA) in recall. It was hypothesized that a delayed recall task and examination of the serial position curve would be a more sensitive test for ear asymmetries than those used in previous studies. In Experiment 1, 30 right-handed male subjects were given lists of words to recall, presented to either the left or right ear, with language chatter, baroque music, or no stimulus concurrently presented to the opposite ear. Both immediate and delayed recall were assessed. The results indicated that the strongest REAs appeared in delayed recall. Moreover, the REAs occurred regardless of the stimulus conditions co-occurring in the other ear. Experiment 2 was done to test whether the REAs in the delayed recall were due to rehearsal biases. Twenty-four right-handed male subjects recalled lists of words with standard instructions and instructions to recall in the reverse order of input (limiting rehearsal). The results indicated that the backward instructions limited and controlled rehearsal and, hence, input to long-term storage; the REAs occurred in delayed recall under all instructional conditions.
\end{abstract}

There is a wealth of evidence that suggests that the two halves of the human brain are specialized in some of their activities. In general, the left hemisphere is involved in language and analytical functions and the right hemisphere in spatial and holistic processing. The technique of dichotic listening, introduced by Broadbent (1956) more than 2 decades ago, has often been used to document the right-ear/left-hemisphere advantage for verbal material (Richardson \& Knight, 1970). However, this method has been widely criticized for a number of reasons.

The evidence from brain-damaged patients in the clinical literature (i.e., Sperry, Gazzaniga, \& Bogen, 1967) suggested striking differences in the information processing capacities of the hemispheres. Yet the differences found in the dichotic listening studies, when in the predicted direction, are small (Freides, 1977). For example, one study (Satz, Achenbach, Pattershall, \& Fennell, 1965) found the right-ear advantage (REA) to be only $4 \%$ to $10 \%$ of the amount recalled in the left ear. Blumstein, Goodglass, and Tartter (1975) contend that the REA is only 6\%. Moreover, two studies (Blumstein et al., 1975; Pizzamiglio, DePascalis, \&

These studies were supported by Grant RO3-MH32886-01 from the National Institute of Mental Health, USDHEW, to the first author. The authors wish to thank Ken Okrent, Steve Lipstein, and Scott Ellington for conducting the test sessions and scoring the data. Thanks are also due to David Freides and Eugene Winograd for help in the original design for the studies and to Eugene Winograd (again) for help in the preparation of the manuscript. Reprint requests should be addressed to John Neil Bohannon III, School of Psychology, Georgia Institute of Technology, Atlanta, Georgia 30332.
Vignatti, 1974) report that over $20 \%$ of their subjects switched ear dominance upon retest.

The reason for these inconsistencies may lie in the confounding of true REA effects and output interference. Many subjects exhibit a preference to report the material directed to the right ear first, before recalling the material to the left ear (Bartz, Satz, Fennell, \& Lally, 1967; Bryden, 1961, 1965). Inglis (1965) argued that since the material directed to the left ear must be held in short-term storage, it is susceptible to output interference (see Tulving \& Arbuckle, 1966). Oxbury, Oxbury, and Gardiner (1968) controlled for the order of ear reported first. They found the material reported first, regardless of ear of input, was recalled better than the material reported later. Freides (1977) replicated these results and found the amount recalled to be correlated with output position. Although some (Satz, 1968) contend the REA exists even when order of ear reported is controlled, evidence from the above studies suggests that dichotic measures of hemispheric asymmetry may only detect response biases.

Monaural techniques, with which to-be-remembered material is presented only to the left or right ear on each trial, avoiding the output interference confounding of the dichotic method, present a mixed picture. Dirks (1964) found no REA with monaural presentation but obtained an REA with the same stimuli when they were presented dichotically. Such findings have led many to argue that ear asymmetries cannot be observed with monaural techniques (Kimura, 1964; Nagafuchi, 1970; Satz, 1968). However, several studies have found asymmetries using monaural techniques under varying conditions (Bakker, 1967; Bakker \& Boeijima, 1970; Bever, 
1971; Frankfurter \& Honeck, 1973). A review of these studies (Bever, Hurlig, \& Handel, 1976) suggests that the more complex and demanding the task, the larger will be the ear asymmetry.

Kimura (1961, 1964, 1967), assuming the accepted hemispheric processing differences stated previously, proposed an explanation of why REAs are detected more frequently under dichotic than under monaural procedures. She argued that the contralateral ear pathways are superior to the ipsilateral neural tracts. Without dichotic stimulus presentation, the ipsilateral tracts can carry information to the appropriate hemisphere, thereby making the right ear as efficient as the left for verbal material. During dichotic presentation, the ipsilateral connections are occluded, forcing the information to travel on the dominant contralateral pathways. When normally lateralized subjects listen to verbal material directed to the left ear under dichotic conditions, they hear a degraded signal that has been forced to travel along the dominant contralateral tract to the right hemisphere and across the corpus callosum to the left hemisphere for processing. More opportunity for interference and decay occurs on this roundabout route, according to Kimura (1967). Although this mechanism is not universally accepted, it is supported by experimental (Studdert-Kennedy, 1975), physiological (Rosenweig, 1957), and clinical (Milner, Taylor, \& Sperry, 1968; Sparks \& Geschwind, 1968) evidence.

The problem with much of the research on right-ear dominance for verbal material may be methodological. Most of the dichotic studies are suspect, due to output interference confounding. It is clear that monaural procedures are preferable but give contrary results.

The point of departure for the research presented here is that most studies, both monaural and dichotic, analyze only total recall of a list of items presented to either ear. But we know that assessment of the serial position curve is a much more sensitive measure. Analysis of the serial position by ear of input could answer additional questions unaddressed by Kimura's (1961) model of ear asymmetries. For example, since recall of verbal items is a frequent dependent measure, where in the recall process does the REA take place? The Bakker and Boeijima (1970) study suggests a possibility. They found a significant REA in total recall only when they required the subjects to recall the items in the order of input. Such instructions enhance the primary part of the serial position curve and output from long-term recall (Craik, 1970). It is possible that degraded verbal information forced to travel the circuitous route from the left ear has difficulty making the transition from short to long term storage. A delayed recall task (Craik, 1970) exclusively assessing the output from long-term memory would test this hypothesis.

The first experiment tested the Kimura (1961) model of ear asymmetry, using a modified monaural listening procedure. To avoid the problems connected with dichotic technique, the items to be recalled were pre- sented to only one ear at a time. Concurrently, in the opposite ear, the subjects were presented one of three stimulus conditions: cocktail party chatter that was discriminably language but incomprehensible, complex melodies of baroque music, or nothing. If Kimura is correct, then some stimulus in the right ear is needed to occlude ipsilateral pathways and cause degradation in the verbal information arriving from the left ear. The use of music as a competing stimulus was necessary to assess the possibility that the effect is specific to competition from exclusively verbal information. Dependent measures were the number of items remembered per serial position of input, for both immediate and delayed recall tasks.

\section{EXPERIMENT 1}

\section{Method}

The design of Experiment 1 was a 2 (ear of input: left or right) by 3 (type of competing stimulus: language chatter, music, or no competing stimulus) by 2 (time of recall: immediate or delayed) by 10 (serial positions of the stimulus lists) withinsubjects factorial design.

The subjects for Experiment 1 were 30 undergraduate male volunteers from psychology classes. The subjects were screened on a version of Bryden's (1965) handedness questionnaire. To be included in the study, all subjects had to report biases to eat, throw a ball, bat, kick, and write with the right limb. In addition, the subjects had no history of sinistrality in their immediate families.

The stimuli for Experiment 1 were 240 single-syllable AA nouns taken from the Thorndike-Lorge (1944) norms. The words were randomily assigned to 24 lists of 10 words each. The lists were then randomly assigned to one of four blocks of six ear/competing stimulus conditions (see Table 1 for a sample block of lists). Thus each ear/competing stimulus condition had four examples. Six such groups of lists were constructed such that each list of 10 words occurred in each ear/competing stimulus condition equally. In addition, the headphones were reversed for half of the subjects, to avoid any possible "equipment" effects. The baroque melodies were recordings from the works of Bach, Scarlatti, and Vivaldi. The language chatter was several human voices overdubbed on audiotape to create a cocktail party chatter effect recognizable as English language but unintelligible to native speakers.

Table 1

Sample Block of Stimulus Material

\begin{tabular}{|c|c|c|}
\hline $\begin{array}{c}\text { Item } \\
\text { Number }\end{array}$ & $\begin{array}{l}\text { Ear of } \\
\text { Input }\end{array}$ & Stimuli \\
\hline 1 & $\begin{array}{l}\text { Left } \\
\text { Right }\end{array}$ & $\begin{array}{l}\text { Baroque Music } \\
\text { Words to Be Recalled (10) }\end{array}$ \\
\hline 2 & $\begin{array}{l}\text { Left } \\
\text { Right }\end{array}$ & $\begin{array}{l}\text { Language } \\
\text { Words to Be Recalled (10) }\end{array}$ \\
\hline 3 & $\begin{array}{l}\text { Left } \\
\text { Right }\end{array}$ & $\begin{array}{l}\text { Words to Be Recalled (10) } \\
\text { Blank }\end{array}$ \\
\hline 4 & $\begin{array}{l}\text { Left } \\
\text { Right }\end{array}$ & $\begin{array}{l}\text { Blank } \\
\text { Words to Be Recalled (10) }\end{array}$ \\
\hline 5 & $\begin{array}{l}\text { Left } \\
\text { Right }\end{array}$ & $\begin{array}{l}\text { Words to Be Recalled (10) } \\
\text { Language Chatter }\end{array}$ \\
\hline 6 & $\begin{array}{l}\text { Left } \\
\text { Right }\end{array}$ & $\begin{array}{l}\text { Words to Be Recalled (1.0) } \\
\text { Baroque Music }\end{array}$ \\
\hline
\end{tabular}


The stimuli were recorded on Ampex tape using a Studer 1500 reel-to-reel tape deck, with the to-be-remembered items spoken at the rate of 1 word $/ \mathrm{sec}$. The tapes were then rerecorded on cassette tape for delivery to the subjects, using headphones and a BIC T-3 cassette deck.

The subjects were tested individually and instructed to recall the 10 words from each group of items regardless of the stimuli occurring in the other ear. Two practice trials were given using 10-word lists similar to the test lists, to familiarize the subjects with the stimuli and procedure. Subjects were not warned of the ear or condition of each list prior to presentation, nor that there was to be a final delayed recall. Following each 10 -word presentation, the subjects were asked to recall orally as many words as possible in any order. When all 24 10-word items had been presented, the subjects were given a piece of paper and asked to write down as many of the words from all the 10 -word items that they could remember.

\section{Results}

The recall protocols were analyzed by a 2 (ear of input) by 2 (recall times) by 3 (competing stimulus conditions) by 5 (blocks of two serial positions of items at input) within-subjects factorial analysis of variance. The lone significant effect of ear of input occurred in the three-way interaction between ear of input, time of recall, and serial position $[F(4,116)=3.16, p<.05]$. This interaction is shown in Figure 1.

A Fisher's least-significant difference (1.s.d.) test revealed no significant $(p<.05)$ REAs in the serial positions of the immediate recall curve. On the other hand, three REAs occurred in the serial positions of the delayed recall curve (see Figure 1). Moreover, the REAs occurred in the initial three blocks of serial positions.

Another finding of note was that the ear effect did not interact with competing stimuli. This is not to say

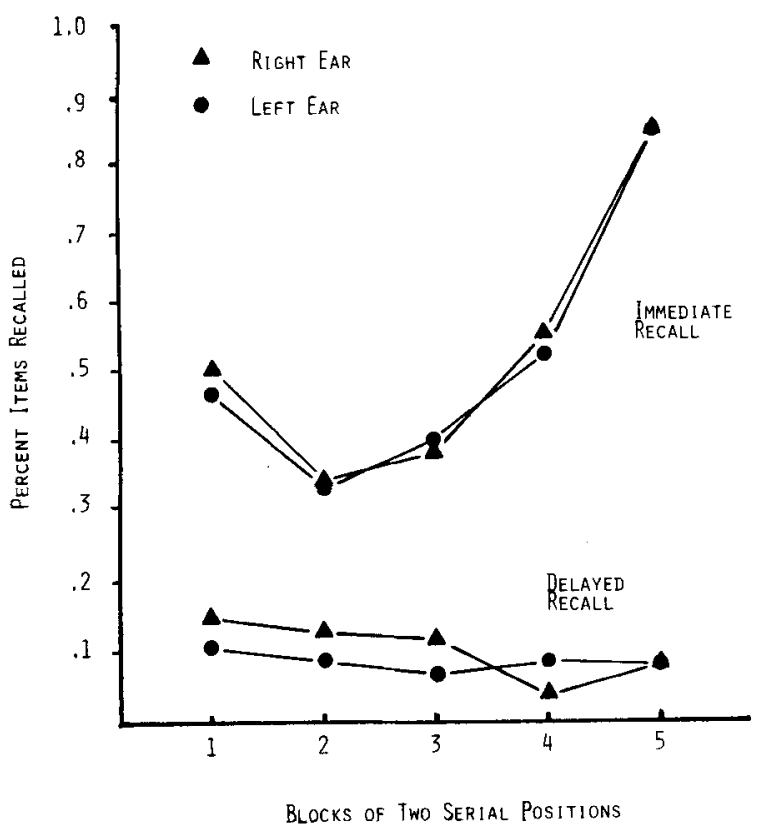

Figure 1. Recall scores from Experiment 1 using two times of recall. The data are collapsed over three types of competing conditions occurring in the opposite ear. that the competing stimuli had no effect; this factor interacted reliably with serial position $[\mathrm{F}(8,232)=1.99$, $\mathrm{p}<.05]$. A multiple comparison of the means (1.s.d.) of this interaction revealed that the chatter condition suppressed the primacy part of the serial position curve.

\section{Discussion}

The most conspicuous finding of Experiment 1 was that REAs can be obtained using monaural techniques if the serial positions at input are noted and recall from long-term store is assessed. The triple interaction among ear, time of recall, and serial position revealed no REAs in immediate recall. This finding replicates other studies (Dirks, 1964; Kimura, 1969) that used monaural techniques and assessed immediate recall. However, significant REAs appeared in the protocols from delayed recall. This suggests that the verbal information arriving from the left ear has more difficulty entering long-term storage than does the material arriving from the right ear. This agrees with the Bakker and Boeijima (1970) result that REAs occur in monaural tasks when the output from long-term storage is enhanced.

However, many (e.g., Atkinson \& Shiffrin, 1968) would argue that immediate recall from the initial serial positions is also output from long-term storage. Why were there no REAs detected in immediate recall in the initial serial positions? This may be due to both the subjects' recall variability and the subtlety of the REA effect. It is possible that although most of the items recalled in the initial serial positions are from long-term storage, some of them may come from shortterm storage. This variability, combined with the subtle nature of the REA in neurologically intact subjects, could have conspired to mask the ear effect in immediate recall. On the other hand, delayed recall would be expected to remove completely the contribution of short-term storage.

Another finding from Experiment 1 was that REAs appeared in delayed recall regardless of the stimulus conditions occurring in the other ear. This finding directly contradicts Kimura's (1969) model of ear asymmetries. She asserted that no REAs occur when verbal information is processed from the left ear when nothing is occupying the right ear's pathways. Yet REAs appeared in delayed recall even when there was no competing stimulus occurring in the right ear. It seems that Kimura's (1969) model must be seriously reconsidered in light of these new data.

But before we can speculate about underlying structural causes of the ear asymmetries in delayed recall, one last problem must be dealt with. It is generally accepted that rehearsal plays a role in the transfer of information to long-term memory ${ }^{1}$ (Atkinson \& Shiffrin, 1968; Craik, 1970). Hence, if the output from delayed recall consists of items in long-term storage, they are also the items that have been rehearsed. It is possible that subjects have a tendency to attend to, rehearse, and process deeply the items arriving from the right ear 
more than the items arriving from the left ear. A set of instructions that controls or limits the processing of items in sort-term storage, possibly inhibiting their transitions to more permanent storage, could test this possibility. If the REAs in delayed recall are due to this tendency, then instructions that restrict processing (e.g., instructions to recall the items backward) should reduce the REAs in delayed recall.

Experiment 2 tested this hypothesis by comparing the REAs in immediate and delayed recall using two instructional sets, standard instructions at input and instructions to recall the items in the reverse order of input. In addition, no competing stimuli were used in the other ear, since Experiment 1 indicated that such a manipulation was not necessary for ear asymmetries to occur.

\section{EXPERIMENT 2}

\section{Method}

The design of Experiment 2 was a 2 (types of instructions at input: standard or recall backward) by 2 (ear of input: right or left) by 2 (time of recall: immediate or delayed) by 10 (serial position of the items at input) within-subjects factorial design.

The subjects for Experiment 2 were 24 undergraduate male volunteers taken from psychology classes. The subjects were screened for handedness and familial history of sinistrality, as in Experiment 1.

The stimuli for Experiment 2 were 200 single-syllable AA nouns taken from the Thorndike-Lorge (1944) norms. The words were randomly assigned to 20 lists of 10 words each. Each list was randomly assigned to two groups of 10 lists (2 practice lists and 8 test lists). The lists were then recorded on either the right or left track of a stereo tape at the rate of 1 word/sec, using the same equipment as that used in Experiment 1 . The final cassettes used for delivery of the stimuli were presented to the subjects such that each 10-word list occurred in each ear/instruction condition equally often. The stimuli were presented to the subjects through headphones, which were reversed for half of the subjects, as in Experiment 1.

The subjects were tested individually. The standard instructions simply required the subject to orally recall as many words as possible immediately following the presentation of each 10-word list. After hearing eight test lists and attempting to recall each list, the subjects were given a piece of paper and asked to write down as many words from all the lists as they could remember. The procedure for the backward instructions was identical, except that the subjects were required to recall the items orally in reverse order from input, last one first. The order of presentation of the instructions was counterbalanced across subjects.

\section{Results}

The number of items recalled was analyzed by a 2 (ears) by 2 (types of instructions) by 2 (times of recall) by 5 (blocks of two-serial positions) withinsubjects factorial analysis of variance.

The differential effects of the backward instructions on recall (collapsed across ear conditions) can be seen in the significant interaction among instruction type, time of recall, and serial position $[F(4,92)=8.91, p<.001]$. This interaction is shown in Figure 2. An 1.s.d. compari-

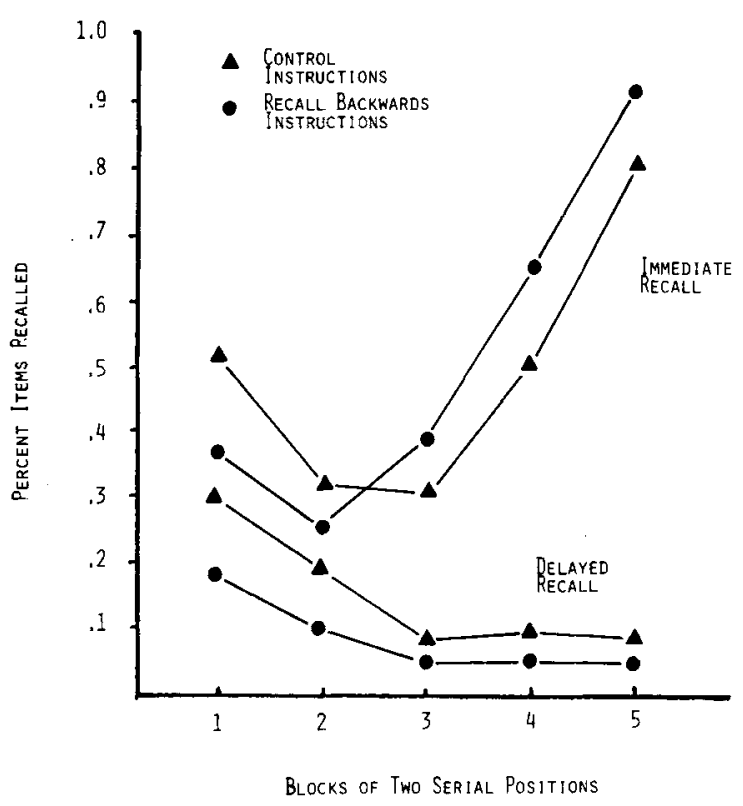

Figure 2. Recall scores from Experiment 2 using two times of recall and two types of instructions at input. The data are collapsed over ear of input.

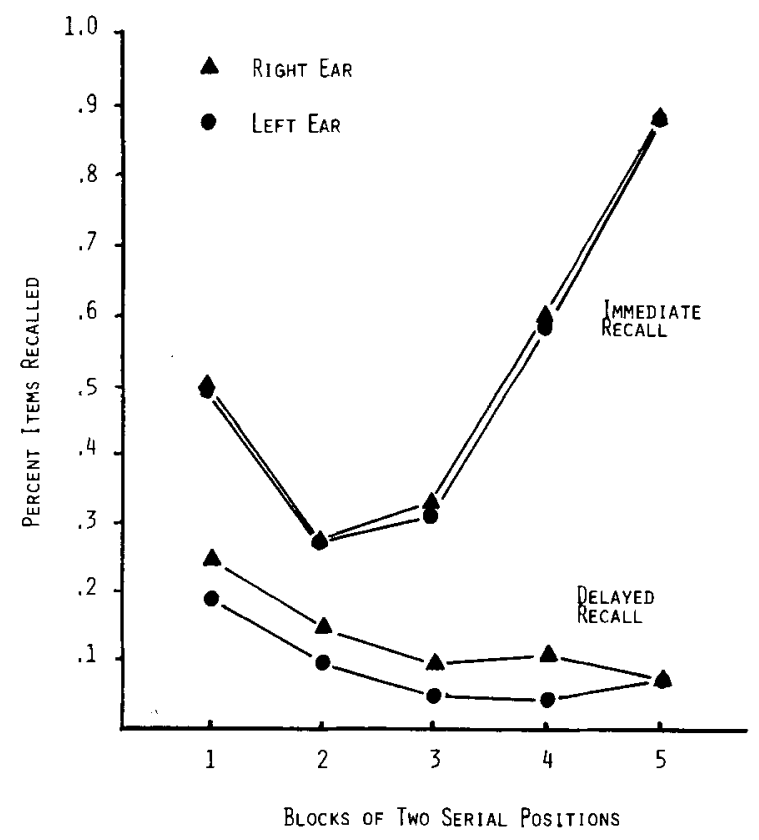

Figure 3. Recall scores from Experiment 2 using two times of recall. The data are collapsed over two types of instruction.

son of the means revealed that the backward instructions enhanced the recency part and depressed the primacy part of the serial position curve derived from immediate recall (see Figure 2). The deleterious effect of backward recall instructions on long-term memory is most clearly seen in the delayed recall data. Delayed recall in the backward condition was severely depressed.

A significant interaction among ear of input, time of 
recall, and serial position $[\mathrm{F}(4,92)=2.47, \mathrm{p}<.05]$ was obtained and can be seen in Figure 3. An l.s.d. comparison of the means in this interaction indicated that the REAs occurred in delayed recall, but not in immediate recall. Moreover, the REAs occurred in delayed recall regardless of instructions (see Figure 3 for ear comparisons collapsed over instructions), since the effect of instructions did not interact with the effect of ear of stimulus input.

\section{Discussion}

It is clear from the results of Experiment 2 that the ear asymmetries found in delayed recall are not simply rehearsal biases. Equally strong REAs appeared in delayed recall protocols even when rehearsal was restricted and controlled by the "recall backward" instructions. Some other mechanism must be causing these asymmetries.

The conflicting results from prior studies of ear asymmetries can now be resolved. When the order of ear reported first is controlled in dichotic procedures, REAs are slight to nonexistent (Freides, 1977; Oxbury et al., 1968). Since these studies assessed only immediate recall (thus mixing output from short- and long-term storage), the lack of asymmetry should be expected. The results of the studies reported here show minimal REA (3\%-6\%) during immediate recall. On the other hand, the REA in delayed recall obtained in the present studies was $20 \%$ to $30 \%$ of the recall from the left ear. It seems that whatever asymmetry of the brain causes an REA, it is most clear when long-term memory alone is assessed.

The contention by Bever et al. (1976) that the REA increases with the difficulty of the task was only partially supported. While it is true that delayed recall is a more difficult task than immediate recall (see Figure 2), it is also true that delayed recall in the backward condition of Experiment 2 was the most difficult of all (see Figure 2). Yet instructional set did not interact with ear of input, and significant REAs appeared in the backward condition also (see Figure 3).

In summary, Experiments 1 and 2 indicate that ear asymmetries exist for verbal information and can be demonstrated using monaural presentation. This effect is strongest when the output from long-term storage is exclusively assessed in a delayed recall task. Moreover, this effect is robust enough not to require a competing stimulus occurring in the opposite ear and to appear when delayed recall is depressed through differing instructions.

\section{REFERENCES}

Atrinson, R. C., \& Shiffrin, R. M. Human memory: A proposed system and its control processes. In K. W. Spence \& J. T. Spence (Eds.), Advances in the psychology of learning and motivation research and theory (Vol. 2). New York: Academic Press, 1968.

BAKKER, D. Left-right differences in auditory perception of verbal and nonverbal material by children. Quarterly Journal of Experimental Psychology, 1967, 19, 334-336.
Bakker, D., \& Boeijima, J. A. Ear order effects on ear asymmetry with monaural stimulation. Neuropsychologia, 1970, 8, 385-396.

Bartz, W. H., Satz, P., Fennell, E., \& Lally, J. R. Meaningfulness and laterality in dichotic listening. Journal of Experimental Psychology, 1967, 74, 204-210.

BEVER, T. G. The nature of cerebral dominance in speech behavior of the child and adult. In R. Huxley \& E. Ingram (eds.), Language acquisition models and methods. New York: Academic Press, 1971.

Bever, T. G., Hurlig, R. R., \& Handel, A. B. Analytic processing elicits right ear superiority in monaurally presented speech. Neuropsychologia, 1976, 14, 175-181.

Blumstein, S., Goodglass, H., \& TartTer, V. The reliability of ear advantage in dichotic listening. Brain and Language, 1975, 2, 226-236.

Broadbent, D. E. Successive responses to simultaneous stimuli. Quarterly Journal of Experimental Psychology, 1956, 8, 145-162.

Bryden, M. P. Order of report in dichotic listening. Canadian Journal of Psychology, 1961, 16, 291-299.

Bryden, M. P. Tachistoscopic recognition, handedness, and cerebral dominance. Neuropsychologia, 1965, 3, 1-8.

Craik, F. I. M. The fate of primacy memory items in free recall. Journal of Verbal Learning and Verbal Behavior, 1970, 9, 143-148.

Dirks, D. Perception of dichotic and monaural verbal material and cerebral dominance for speech. Acta Otolaryngologica, $1964,58,73-80$.

Frankfurter, A., \& Honeck, R. P. Ear differences in the recall of monaurally presented sentences. Quarterly Journal of Experimental Psychology, 1973, 25, 138-146.

Freides, D. Do dichotic listening procedures measure lateralization of information processing or retrieval strategy? Perception \& Psychophysics, 1977, 21, 259-263.

Glanzer, M., \& Koppenaal, L. The effect of encoding tasks on free recall: Stages and levels. Journal of Verbal Learning and Verbal Behavior, 1977, 16, 21-28.

INGLIS, J. Dichotic listening and cerebral dominance. Acta Otolaryngologica, 1965, 60, 231-237.

Kimura, D. Cerebral dominance and the perception of verbal stimuli. Canadian Journal of Psychology, 1961, 15, 166-171.

Kimura, D. Left-right differences in the perception of melodies. Quarterly Journal of Experimental Psychology, 1964, 16, 335-338.

Kimura, D. Functional asymmetry of the brain in dichotic listening. Cortex, 1967, 3, 163-178.

Kimura, D. Spatial localization in left and right visual fields. Canadian Journal of Psychology, 1969, 23, 445-458.

Milner, B., Taylor, L., \& Sperry, R. W. Lateralized suppression of dichotically-presented digits after commissured section in man. Science, 1968, 161, 184-185.

NaGAfuchi, M. Development of dichotic and monaural hearing abilities in young children. Acta Otolaryngologica, 1970, 69, 409-415.

Oxbury, S., Oxbury, J., \& Gardiner, J. Laterality effects in dichotic listening. Nature, 1968, 214, 742.

Pizzamiglio, L., DePascalis, C., \& Vignatti, A. Stability of dichotic listening test. Cortex, 1974, 10, 203-205.

Richardson, D. H., \& KNIGHT, K. M. A bibliography in dichotic listening. Cortex, 1970, 6, 236-240.

Rosenweig, M. R. Representation of the two ears at the auditory cortex. American Journal of Physiology, 1957, 167, 147-158.

SAtz, P. Laterality effects in dichotic listening: A reply. Nature, $1968,218,277-278$.

Satz, P., Achenbach, L., Pattershall, E., \& Fennell, E. Order of report, ear asymmetry, and handedness in dichotic listening. Cortex, 1965, 1, 377-396.

Sparks, R., \& Geschwind, N. Dichotic listening in man after section of neocortical commissures. Cortex, 1968, 4, 3-16.

Sperry, R. W., Gazzaniga, M. S., \& Bogen, J. E. Inter- 
hemispheric relationships: The neocortical commissures, syndromes of hemisphere disconnection. In P. J. Vinken \& J. M. Sprague (Eds.), Handbook of clinical neurology (Vol. 4). Amsterdam: North Holland, 1967.

Studdert-Kennedy, M. Two questions. Brain and Language, 1975, 2, 123-130.

Thorndike, E. L., \& LoRge, I. The teacher's word book of 30,000 words. New York: Columbia University Press, 1944.

Tulving, E., \& ARbuckle, T. Y. Input and output interference in short-term associative memory. Journal of Experimental Psychology, 1966, 72, 145-150.

\section{NOTE}

1. It is convenient for us to discuss their research in terms of dual-store theory. Other formulations, such as the depth-ofprocessing framework, could have been used instead. Glanzer and Koppenaal (1977) have commented on how these alternatives are at least partly a matter of taste.

(Received for publication August 7, 1980; revision accepted October $8,1980$. 